\title{
An Inequality About Factors of Polynomials
}

\author{
By M. Mignotte
}

Abstract. A sharp inequality is proved about the product of some roots of a polynomial. It is used to bound the height of the factors of a polynomial. Applications are given to the problem of factorization and numerical examples show that these bounds strongly improve the previous ones. we put

I. Introduction. If $R=\Sigma_{j=0}^{d} c_{j} X^{j}$ is a polynomial with complex coefficients,

$$
\|R\|=\left(\sum\left|c_{j}\right|^{2}\right)^{1 / 2}, \quad L(R)=\sum\left|c_{j}\right|, \quad H(R)=\cdot \max \left|c_{j}\right|
$$

We shall first prove:

THEOREM 1. Let $P=\Sigma_{i=0}^{d} a_{i} X^{i}$ be a polynomial with complex coefficients. Let $z_{1}, z_{2}, \cdots, z_{k}$ be those zeros of $P$ (counted with their multiplicities), such that $1 \leqslant\left|z_{1}\right| \leqslant\left|z_{2}\right| \leqslant \cdots \leqslant\left|z_{k}\right|$. Then

$$
\left|a_{d}\right| \prod_{i=1}^{k}\left|z_{i}\right| \leqslant\|P\| .
$$

This inequality improves a result of Mahler [1] who obtained $L(P)$ instead of $\|P\|$ on the right-hand side.

THEOREM 2. Let $Q$ be a polynomial with rational integer coefficients. If $Q_{1} \cdots Q_{m} R=Q$, where $Q_{1}, \cdots, Q_{m}, R$ are polynomials with rational integer coefficients, then

$$
\prod_{j=1}^{m} L\left(Q_{j}\right) \leqslant 2^{D}\|Q\|, \text { where } D=\sum_{j=1}^{m} \operatorname{deg}\left(Q_{j}\right),
$$

and, if for example $Q_{1}=b_{0}+b_{1} X+\cdots+b_{1} X^{1}$, then

$$
\left|b_{i}\right| \leqslant\left(\begin{array}{l}
1 \\
i
\end{array}\right)\|Q\| \text {. }
$$

(This result also holds for Gaussian integer coefficients.)

These inequalities can be used in the theory of transcendental numbers, but we

Received June 28, 1973.

AMS (MOS) subject classifications (1970). Primary $12 \mathrm{D} 05$.

Key words and phrases. Inequality, roots of polynomial, factorization.

Copyright $\bigcirc$ 1974, American Mathematical Society 
shall not speak of this here. They are also useful in the problem of factorization of polynomials over $\mathbf{Z}$ as we shall see now.

We recall the method of $\mathrm{H}$. Zassenhaus [3]. Put

$$
F(X)=X^{n}+a_{1} X^{n-1}+\cdots+a_{n}, \quad a_{i} \in \mathbf{Z},
$$

and assume that

$$
G(X)=X^{m}+b_{1} X^{m-1}+\cdots+b_{m}, \quad b_{j} \in \mathbf{Z}, m \leqslant n / 2,
$$

is a factor of $F$.

Suppose that we find $M$ such that for any such $G$ we have $H(G) \leqslant M$. We take a prime number $p$, not dividing the discriminant of $F$, and choose $r$ such that $p^{r}>2 M$. Then, starting with a factorization into monic polynomials

$$
F \equiv F_{1} \cdots F_{k} \quad(\bmod p),
$$

we get, with the help of Hensel's lemma, well-defined $\bar{F}_{i} \in \mathbf{Z}(X)$ such that

$$
F \equiv \bar{F}_{1} \cdots \bar{F}_{k}\left(\bmod p^{r}\right), \quad \text { with } \bar{F}_{i} \equiv F_{i}(\bmod p), \quad i=1, \cdots, r,
$$

and such that the coefficients of the $\bar{F}_{i}$ belong to the interval $\left.]-p^{r} / 2, p^{r} / 2\right]$.

It is now clear that we are able to factorize $F$ over $Z$. The problem is now to find a value for $M$.

Zassenhaus remarked that, if $|z| \leqslant A$ for any root $z$ of $F$, then

$$
\left|b_{j}\right| \leqslant\left(\begin{array}{c}
m \\
j
\end{array}\right) A^{j}
$$

It is well known that we can take

$$
A=\max \left|a_{i}\right|+1 .
$$

Zassenhauss also used the bound

$$
A=\max _{1 \leqslant i \leqslant n}\left|\frac{\left|a_{i}\right|}{\left(\begin{array}{c}
n \\
i
\end{array}\right)}\right|^{1 / i} /\left(2^{1 / n}-1\right) .
$$

To show the strength of (2), we take two examples given in [4] to compare (3) and (4).

Put

$$
F_{1}(X)=X^{15}+30 X^{14}+5 X^{13}+2 X^{12}+5 X+2
$$

and

$$
F_{2}(X)=X^{8}+8 X^{7}+21 X^{6}+21 X^{5}+42 X^{4}+13 X^{3}+12 X^{2}-14 X+12 .
$$

For $F_{1}$, we get 


$$
\begin{array}{ll}
M_{1} \leqslant 2.8 \cdot 10^{10} & \text { by (3), } \\
M_{1} \leqslant 2.7 \cdot 10^{9} & \text { by (4), }
\end{array}
$$

and, for $F_{2}$,

$$
\begin{array}{ll}
M_{2} \leqslant 3.5 \cdot 10^{6} & \text { by (3), } \\
M_{2} \leqslant 1.4 \cdot 10^{5} & \text { by (4); }
\end{array}
$$

whereas (2) gives

$$
M_{1} \leqslant 1083 \text { and } M_{2} \leqslant 348 .
$$

(In fact, $F_{1}$ is irreducible: Rouche's theorem shows that all its roots but one lie in the disk $|z|<1$.)

II. Proof of Theorem 1. A proof can be found in [2], but we prefer to deduce it from the following elementary lemma which gives a stronger result.

Lemma 1. Let $P(X)$ be a polynomial with complex coefficients and $\alpha$ be a nonzero complex number. Then

$$
\|(X+\alpha) P(X)\|=|\alpha|\left\|\left(X+\bar{\alpha}^{-1}\right) P(X)\right\| .
$$

Proof. Write

$$
\begin{aligned}
& P(X)=\sum_{k=0}^{m} a_{k} X^{k} \\
& Q(X)=(X+\alpha) P(X)=\sum_{k=0}^{m+1}\left(a_{k-1}+\alpha a_{k}\right) X^{k}, \\
& R(X)=\left(X+\bar{\alpha}^{-1}\right) P(X)=\sum_{k=0}^{m+1}\left(a_{k-1}+\bar{\alpha}^{-1} a_{k}\right) X^{k},
\end{aligned}
$$

with $a_{-1}=a_{m+1}=0$.

Then

$$
\|Q\|^{2}=\sum_{k=0}^{m+1}\left|a_{k-1}+\alpha a_{k}\right|^{2}=\sum_{k=0}^{m+1}\left(a_{k-1}+\alpha a_{k}\right) \overline{\left(a_{k-1}+\alpha a_{k}\right)}
$$

which expands to

$$
\sum_{k=0}^{m+1}\left(\left|a_{k-1}\right|^{2}+\alpha a_{k} \bar{a}_{k-1}+\bar{\alpha} a_{k-1} \bar{a}_{k}+|\alpha|^{2}\left|a_{k}\right|^{2}\right) .
$$

Expanding $|\alpha \cdot|^{2}\|R\|^{2}$ yields the same sum. 
Thus we have $\|Q\|=|\alpha|\|R\|$, which proves the lemma.

Lемма 2. Let $x_{1}, x_{2}, \cdots, x_{m}$ be complex numbers,

$$
0<\left|x_{1}\right| \leqslant \cdots \leqslant\left|x_{q}\right|<1 \leqslant\left|x_{q+1}\right| \leqslant \cdots \leqslant\left|x_{m}\right|, \quad q \geqslant 0 .
$$

Put

$$
\begin{aligned}
& S(X)=\left(X-x_{1}\right) \cdots\left(X-x_{m}\right) \\
& T(X)=\left(X-\bar{x}_{1}^{-1}\right) \cdots\left(X-\bar{x}_{q}^{-1}\right)\left(X-x_{q+1}\right) \cdots\left(X-x_{m}\right) .
\end{aligned}
$$

Then

$$
\|S\|=\left|x_{1} \cdots x_{q}\right|\|T\| .
$$

Proof. By induction on $q$. For $q=0,(5)$ holds. Assume $q>0$ and put

$$
\bar{S}(X)=S(X) /\left(X-x_{1}\right), \quad \bar{T}(X)=T(X) /\left(X-\bar{x}_{1}^{-1}\right) .
$$

Then

$$
\begin{aligned}
\|S\| & =\left\|\left(X-x_{1}\right) \bar{S}(X)\right\|=\left|x_{1}\right|\left\|\left(X-\bar{x}_{1}^{-1}\right) \bar{S}(X)\right\| \quad \text { (by Lemma 1) } \\
& =\left|x_{1}\right|\left|x_{2} \cdots x_{q}\right|\left\|\left(X-\bar{x}_{1}^{-1}\right) \bar{T}(X)\right\| \quad \text { (by induction hypothesis) } \\
& =\left|x_{1} \cdots x_{q}\right|\|T\| .
\end{aligned}
$$

This implies the following refinement of Theorem 1 .

Proposition. Let $P(X)=a_{m} X^{m}+\cdots+a_{0}=a_{m}\left(X-x_{1}\right) \cdots\left(X-x_{m}\right)$ where $x_{1}, \cdots, x_{m}$ are complex numbers such that

$$
\left|x_{1}\right| \leqslant \cdots \leqslant\left|x_{q}\right|<1 \leqslant\left|x_{q+1}\right| \leqslant \cdots \leqslant\left|x_{m}\right|, \quad q \geqslant 0 .
$$

Then

$$
\|P\|^{2} \geqslant\left|a_{m}\right|^{2}\left|x_{q+1} \cdots x_{m}\right|^{2}+\left|a_{0}\right|^{2}\left|x_{q+1} \cdots x_{m}\right|^{-2} .
$$

Proof. Put

$$
Q(X)=a_{m} \prod_{i=1}^{q}\left(X-\bar{x}_{1}^{-1}\right) \prod_{i=q+1}^{m}\left(X-x_{i}\right)=b_{m} X^{m}+\cdots+b_{0}
$$

First assume $x_{1} \neq 0$. Then by Lemma $2,\|P\|=\left|x_{1} \cdots x_{q}\right|\|Q\|$, hence

$$
\|P\|^{2} \geqslant\left|x_{1} \cdots x_{q}\right|^{2}\left(\left|b_{m}\right|^{2}+\left|b_{0}\right|^{2}\right),
$$

from which the result follows.

If $x_{1}=\cdots=x_{n}=0(n \leqslant q)$, then $a_{0}=0$, so we just have to prove $\|P\|^{2} \geqslant\left|a_{m}\right|^{2}\left|x_{q+1} \cdots x_{m}\right|^{2}$. But, in fact, replacing $P(X)$ by $P(X) / X^{n}$ in the above argument yields the stronger result 


$$
\|P\|^{2} \geqslant\left|a_{m}\right|^{2}\left|x_{q+1} \cdots x_{m}\right|^{2}+\left|a_{n}\right|^{2}\left|x_{q+1} \cdots x_{m}\right|^{-2} .
$$

Remarks. (1) The proof of Theorem 1 is quite elementary while the previous inequalities were weaker and based on transcendental results such as Jensen's or Parseval's formula. We leave an analytic proof of Lemma 2 as exercise to the reader.

(2) In a certain sense, Theorem 1 is the best possible: the inequality is not always true if we replace $\|P\|$ by $\left(\Sigma\left|a_{j}\right|^{e}\right)^{1 / e}$ for $e>2$. (Take for example $P(X)=$ $X^{2}-2 a X-1$ where $a$ is a sufficiently large positive number.)

III. Proof of Theorem 2. The well-known expression of the coefficients of a polynomial gives:

Lemma 3. Let $P$ be as in Theorem 1. Then

$$
\left|a_{i}\right| \leqslant\left(\begin{array}{c}
d \\
i
\end{array}\right)\left|z_{1} \cdots z_{k}\right|\left|a_{d}\right|
$$

and

$$
\sum_{i=0}^{d}\left|a_{i}\right| \leqslant 2^{d}\left|z_{1} \cdots z_{k}\right|\left|a_{d}\right| .
$$

The theorem follows easily from Lemma 3 and Theorem 1.

Acknowledgement. I would like to thank the referee for several helpful comments.

Université Paris-Nord

Département de Mathématiques

Centre Scientifique et Polytechnique

Place du 8 Mai 1945

93 Saint-Denis 93206, France

1. K. MAHLER, “An application of Jensen's formula to polynomials," Mathematika, v. 7, 1960, pp. 98-100. MR 23 \#A1779.

2. M. MIGNOTTE, "Critères d'irréducibilité des polynomes sur un corps de nombres," Enseignement Math., v. 18, 1972, pp. 191-200.

3. H. ZASSENHAUS, “On Hensel factorization. I," J. Number Theory, v. 1, 1969, pp. 291 311. MR $39 \# 4120$.

4. H. G. ZIMMER, Computational Problems, Methods and Results in Algebraic Number Theory, Lecture Notes in Math., vol. 269, Springer-Verlag, Berlin, 1972. 